\title{
Usos de las TIC en América Latina: una caracterización
}

\author{
Uses of ICT in Latin America: a characterization \\ Darío J. Quiroga-Parra ${ }^{1 *} \quad$ Joan Torrent-Sellens ${ }^{2} \quad$ Claudia Patricia Murcia Zorrilla $^{1}$ \\ Recibido 20 de febrero de 2015, aceptado 2 de junio de 2016 \\ Received: February 20, 2015 Accepted: June 2, 2016
}

\begin{abstract}
RESUMEN
Las tecnologías de la información y la comunicación-TIC generaron un dinamismo productivo, económico y social en los países desarrollados, desde las pasadas cuatro décadas, con la denominada revolución tecnológica digital e industrial. Estos hechos, transformaron a la par los procesos de innovación, de producción y los mercados globales. Fenómeno observado en los contextos macro y microeconómicos. El objetivo de este documento consistió en un análisis estadístico del estado real y uso de las TIC en América Latina en tres períodos de los años dos mil. La metodología utilizada fue la ANOVA con varianza simple y medias, con la prueba F. El uso de factoriales y clústeres de manera sistemática fueron las técnicas manejadas, en estudios transversales, con variables proxis, para una muestra de veintidós países, en una población de treinta y dos. Los resultados mostraron el comportamiento real de las TIC en clústeres diferenciados, destacándose sinergias económicas potenciales entre los países estudiados. El trabajo concluyó sugiriendo que, en América Latina el uso y aplicación de las TIC se mostró aún marginal para la mayoría de los países, hechos contrastados frente a estudios previos y literatura internacional existente, en las estructuras macro y microeconómicas de países desarrollados.
\end{abstract}

Palabras clave: TIC, América Latina, productividad, paradoja TIC, paradoja productividad, innovación.

\begin{abstract}
The information communication technologies - ICT generated a productive, economic and social dynamism through developed countries for the last four decades, with the digital technological and industrial revolution. These facts, transformed the process of innovation, production and global markets. Phenomenon observed in the macro and micro contexts. The aim of this document was a statistical analysis that shows tendencies in the use of ICT in Latin America in three different periods during the first decade of the twentieth century. The methodology used was ANOVA with simple variance and statistical average with test F. At same time, the factorials and clusters were the techniques handled in cross-sectional studies with variable proxies; it was a sample for twenty-two countries, out of thirty-three. The results showed the actual behavior of ICT in different clusters, highlighting potential economic synergies among the countries studied. The work was completed showing that in Latin America the use and application of ICT is still marginal for most countries, these evidences were contrasted with previous studies and current international literature on the macro and microstructures of developed countries.
\end{abstract}

Keywords: ICT, Latin America, productivity, ICT paradigm, productivity paradox, innovation.

1 Facultad de Ciencias Administrativas, Económicas y Contables. Universidad Cooperativa de Colombia (UCC). Colombia. E-mail: dario.quirogap@campusucc.edu.co; claudia.murcia@campusucc.edu.co

2 Business Studies and Internet Interdisciplinary Institute (IN3). Universidad Oberta de Catalunya (UOC). España. E-mail: jtorrent@uoc.edu

* Autor de correspondencia 


\section{INTRODUCCIÓN}

Las tecnologías de la información y comunicación (TIC) desde las últimas cuatro décadas están impulsando un proceso de transformación tecnológica y de generación de nuevo conocimiento e innovación sin precedentes, en la historia de la humanidad. De manera que, su uso y aplicación científica y empresarial intensiva, dinamizan la transformación tecnológica y de innovación en los países desarrollados. Resultados que se observan en la aceleración de la globalización, en los contextos económicos, sociales y los mercados [17-18, 20-21, 31, 35].

Es así como, los primeros resultados empíricos propician múltiples trabajos académicos de investigación, en diferentes espacios internacionales. Es más, su permanente dinamismo mundial, continúa facilitando importantes debates académicos, en razón de las numerosas evidencias empíricas de su impacto, tanto en la innovación empresarial y la productividad, como en la generación de conocimiento científico. Elementos que hacen ver el surgimiento de un nuevo tipo de economía centrada en el uso intensivo del conocimiento [6-7].

De hecho, esta tecnología en el período comprendido entre 1970 hasta la fecha, ha estado cimentando un sustrato físico y tecnológico mundial, sin precedentes históricos en los países que hacen uso de esta tecnología digital. A la par de la infraestructura tecnológica per se, estas se están consolidando como una tecnología de uso general y transversal, erigiéndose en un paradigma tecnoeconómico y como la columna vertebral de una nueva revolución industrial [5, 16, 19, 49].

No obstante, de los avances tecnológicos digitales en el entramado global, preexisten regiones del mundo que no están absorbiendo y apropiando esta tecnología en sus procesos productivos, científicos y económicos. Ciertamente, América Latina (AL) muestra ser una de ellas. De hecho, el documento plantea la hipótesis que, los niveles de uso de TIC en la región son marginales, por lo que, el objetivo de trabajo es conocer estadísticamente el estado real de esta tecnología en la región latinoamericana en los períodos 2000, 2006 y 2008.

Metodológicamente, el trabajo hace uso de la ANOVA con varianza simple y las medias aritméticas, utilizando la prueba F. Como técnicas centrales se utilizan: el factorial y los clústeres de manera sistemática. Se tomó una muestra de veintidós países de AL, de una población de treinta y dos, en análisis de cortes transversales, utilizando variables proxis.

Sistemáticamente, el artículo en su primera parte presenta una breve revisión de la literatura del tema. Le siguen en su orden la metodología empleada, los resultados empíricos logrados y la discusión académica. Al final del documento, se muestran las conclusiones y las referencias bibliográficas.

Los resultados expresan abiertamente el desempeño del uso de la TIC por medio de conglomerados diferenciables y la existencia de sinergias y correlaciones entre economías de la región. El artículo concluye señalando que, América Latina presenta una ineficiencia en el avance y uso de las TIC frente a la literatura internacional macro y microeconómica existente.

\section{REVISIÓN DE LA LITERATURA}

\section{Las tecnologías de la información y la comunicación}

Los países desarrollados y sus organizaciones empresariales han tenido una subyacente dinámica económica y social desde la aparición y dispersión global de las tecnologías de la información y la comunicación (TIC), reflejada en la innovación y niveles de productividad. Fue así como, a partir de finales de la década de los años noventa, el aceleramiento de los indicadores de productividad y los financieros, producto del uso de las TIC, inicialmente propiciaron un debate académico, en razón de los cambios observados [10, 26-27, 38, 43]. Debate que, se dirimió solo alrededor de los años dos mil [33, 39, 41] con la presencia de nuevas evidencias empíricas de múltiples trabajos en este campo. De hecho, la controversia inicial se dio por la carencia de elementos estadísticos que mostraran las razones causa-efecto entre los indicadores de la eficiencia productiva y su relación con el uso de las TIC.

Al respecto, el trabajo de Brynjolfsson [11] es considerado un estudio pionero en este campo con evidencias empíricas, le siguieron Brynjolfsson y Hitt [13], Bresnahan, Brynjolfsson y Hitt [12]. Hallazgos que permitieron rebatir la paradoja de 
Solow [16, 37] con datos, evidencias empíricas, positivas que la cuestionaron, señalando una explicación del porque la existencia de una relación TIC-productividad. Pero el avance más relevante de este proceso fue la evolución de la literatura de los modelos neoclásicos del crecimiento económico. Así como, la disponibilidad de datos a nivel empresarial que han permitido el estudio de la relación TIC-productividad.

Fue así como, en la década del año dos mil, prosiguieron trabajos microeconómicos (empresariales) como los de Brynjolfsson y Hitt [15], Hempell [24-25], Black \& Lynch [8], Arvanitis [3] y Arvanitis \& Loukis [4]. Estos estudios fueron más allá de la misma incidencia de la TIC en la productividad, a considerar otras variables complementarias. Asimismo, desde la perspectiva macroeconómica otros estudios se fueron dando de manera paralela, desde Jorgenson [27-28], pasando por Timmer, Ypma y Van Ark [44-15], Jorgenson y Vu, [29-30], Van Reenen, Draca, Sadun [48] hasta Quiroga-Parra [40], acrecentando las evidencias empíricas y la literatura internacional sobre el tema.

Inicialmente, los primeros estudios sobre el tema se dieron en Estados Unidos, estos fueron mostrando la importancia de las TIC, tanto en las empresas como en contextos macro-económicos. De hecho, mostraron el incremento de la productividad, dada la incidencia de las TIC sobre los procesos productivos $[26,38]$.

De manera paralela lo fue haciendo Europa, señalando la relevancia productiva y económica de las TIC, en esa región. Así, la Unión Europea [47] resalta varios aportes de estas tecnologías a su economía, como; primero, sugieren que las TIC están cambiando las condiciones de competitividad en sus mercados, en razón del surgimiento de una nueva estructura de mercado local y global. Así como la desaparición de barreras en los canales logísticos, para la entrega de productos y servicios. Segundo, por la incidencia de estas tecnologías digitales sobre los incrementos de innovación. En concreto, se observa la creación de nuevos modelos de negocios, mayor fluidez del manejo de los clientes y una mejora de la calidad de los servicios. Así como la aplicación de nuevas prácticas organizativas proporcionadas por las TIC en el lugar de trabajo que facilitan y aceleran la innovación de productos y procesos [3].
Justamente, los diferentes estudios internacionales por medio del tiempo, han ido mostrando el papel de las TIC en los procesos productivos. Así que, uno de los roles de esta tecnología es el de un nuevo tipo de capital input, especialmente el de la información. Ella ingresa a los procesos productivos transformándolos; y a la par mostrándose estrechamente relacionadas con los procesos generales de innovación. Es así como, Hempell [24] plantea que las TIC en su rol empresarial se están presentando como un prerrequisito en las empresas y países, para poder hacer frente e incursionar en la economía del conocimiento mediante los procesos de innovación, centrados en el uso intensivo del conocimiento. De hecho, el autor plantea que las TIC son una herramienta tecnológica que no trabaja sola, debido a que es un factor complementario al capital humano y al lugar de trabajo (prácticas organizativas).

En la denominada economía del conocimiento, los inputs son los elementos tangibles o intangibles predominantemente, que entran a un proceso productivo, para ser transformados en nuevos productos. Los más relevantes son: Las TIC, el conocimiento, el lugar de trabajo o prácticas organizativas y la innovación.

De este modo, la literatura reciente de frontera sobre el tema ha ido dando reconocimiento acentuado a la existencia de altos grados de complementariedad (coinnovación). Los estudios de coinnovación se han venido dando entre el denominado capital TIC, el capital humano y las nuevas prácticas organizativas (lugar de trabajo) [4]. En donde, a su vez, la innovación genera una incidencia positiva en la productividad empresarial. Es destacable aquí la contribución del capital humano (conocimiento) en la complementariedad con las TIC, elemento que es reconocido ampliamente en los estudios empresariales. Como ejemplo están los nuevos diseños de trabajo y la voz del empleado [8-9]. De manera que, la complementariedad es ahora el nuevo paradigma de las empresas en la emergente economía [4].

De hecho, las evidencias empíricas han venido señalando que, buena parte de la explicación de la productividad de las empresas depende de la dotación de los factores productivos, de la eficiencia y de cómo estos son combinados. En donde las TIC 
y su grado de relación con la calidad del capital humano-conocimiento, y las prácticas organizativas en el lugar de trabajo juegan un rol preponderante.

En síntesis, el surgimiento de las TIC ha venido incidiendo en los procesos de productividad, propiciando a su vez la aparición de unos nuevos factores de producción que, en su complementariedad están elevando los niveles de innovación y de productividad $[4,15,46]$.

\section{Las TIC, una revolución tecnológica y del conocimiento}

Las TIC aparecieron con la invención del transistor que se convirtió en la fuerza impulsora de estas tecnologías digitales. El transistor opera como un interruptor eléctrico y codifica la información de manera binaria. La utilización masiva y la popularización de las TIC se dieron ciertamente con la caída de los precios de este dispositivo central [27], aspecto que está correlacionado en la denominada ley de Moore, y su acelerada expansión con la ley de Metcalfe.

De hecho, el hombre durante toda su existencia ha ido de la mano del conocimiento y de la tecnología. De manera que, la historia tecnológica de la humanidad muestra haber pasado por al menos tres revoluciones relacionadas con el avance en el conocimiento y el desarrollo industrial; sin tener en cuenta aquellas ocurridas durante las eras previas. Estas revoluciones a su vez han ido paralelas a los procesos de mundialización, en la medida en que las comunicaciones, en sus diferentes formas avanzaban. Así, la primera revolución industrial y tecnológica ocurrió a finales del siglo XVIII y comienzos del siglo XIX. Una de sus características centrales fue la sustitución de las herramientas manuales del hombre por máquinas. Ejemplos de estos inventos fueron: la máquina de vapor, la máquina hiladora para diferentes usos, y el diseño del proceso de corte de metales. Igualmente surgió el uso intensivo del carbón, del hierro, y el desarrollo del ferrocarril.

La segunda revolución tecnológica e industrial, ocurrida posterior a 1850 , se puede vincular con el proceso de invención del motor de combustión interna, la energía eléctrica, el diseño y fabricación del automóvil y el avión. A la par surgió la química basada en las ciencias, el sistema eficiente de fundición de acero, entre otros. Simultáneamente fue el inicio de las tecnologías de la información y la comunicación, como el telégrafo y el teléfono.

Para Mokyr [34] citado en Castells [17] estas dos revoluciones muestran algunas características similares en lo observado a partir de 1850 . Pero asimismo, presentan diferencias críticas por ejemplo el uso intensivo del conocimiento científico para producir y orientar el desarrollo tecnológico.

Los historiadores de la ciencia y la tecnología también registran una tercera revolución tecnológica e industrial centrada en el uso de las tecnologías digitales TIC, cuyo eje central se observó alrededor de finales de los años sesenta e inicios de los años setenta, en los Estados Unidos. Dicha revolución tecnológica ha estado centrada en la microelectrónica, las computadoras y las telecomunicaciones. A pesar que su difusión se inició en la década de 1970, sus raíces se remontan a un siglo atrás, en 1806 con la invención del teléfono de Bell.

Otros inventos como el radio de Marconi en 1898, del tubo al vacío de De Forest en 1906 aportaron a este proceso, sin descartar los primeros avances tecnológicos de la Segunda Guerra Mundial, como por ejemplo el primer ordenador programable. En donde ciertamente el transistor, antes mencionado fue uno de los elementos centrales de esta nueva revolución; debido a que es considerado el núcleo central de este cambio tecnológico [17]. Elemento que fue inventado en 1947 por los físicos Bardeen, Brattain y Shockley, en los laboratorios de Bell Murria Hill. La característica de este dispositivo tecnológico fue la generación de dos impulsos eléctricos a mayor ritmo y de modo binario, esto es el de "interrupción y el de paso".

En donde, los protagonistas centrales de las dos primeras revoluciones tecnológicas fueron los países occidentales, precedidos por Europa y liderados por Inglaterra, observándose una incuestionable supremacía occidental anglosajona-alemana-francesa. Sin embargo, los países orientales y en concreto China fueron tecnológicamente superiores durante buena parte de la historia, especialmente durante el renacimiento occidental, a la par que, lo fue la civilización musulmana en el mediterráneo y África en la llamada época de la modernidad [17]. De hecho, el período 1300-1800 para China los historiadores lo consideran de estancamiento [17, 34]. 
No obstante, la segunda revolución industrial cambió de hecho su dirección hacia países como Alemania y Estados Unidos. En donde se dieron los principales avances como la química, la electricidad y la telefonía [17, 35].

La denominada tercera revolución tecnológica digital, acción aún no dimensionada internacionalmente por sus características de información, comunicación y uso intensivo del conocimiento tuvo como epicentro los Estados Unidos, en Silicón Valley. Desde donde rápidamente buena parte de los países industrializados se adhirieron, aportando a partir de múltiples perspectivas tecnológicas.

Es más, sistemáticamente la creación e innovación del microprocesador en 1971 y de la microelectrónica fueron, de hecho, los protagonistas de la revolución de revoluciones, de la actual sociedad global que estamos viviendo. El microprocesador fue inventado por Ted Hoff, este consistió en algo así como en la acción de tener el ordenador en un chip. De hecho, fue uno de los grandes saltos de la humanidad en lo que respecta a la innovación de la microelectrónica y de los computadores [17]. Esta innovación produjo una gran capacidad de integración centrada en el uso de los chips, una de sus características son su tamaño y su capacidad de memoria, medida en millones de megabites (MB) e indicada por la capacidad DRAM (Dinamic Random Acces Memory).

En este sentido, el sinnúmero de inventos e innovaciones de los siglos previos, la acción de "poder haber colocado un ordenador dentro de un chip" "17] impactó al mundo, con una nueva realidad tecnológica. De manera que, la primera caja de cálculo denominada Altair en 1971 fue diseñada, desarrollada y creada por el ingeniero Ed Roberts. Altair fue la base para que los microempresarios Steve Wozniak y Steve Jobs, en una empresa de garaje en Silicón Valley diseñaran y desarrollaran la primera computadora Apple. De hecho, este fue el primer microordenador comercial, y salió al mercado en 1976 de manera exitosa y es considerado históricamente como el ícono fundador de la era de la información. Al Apple como computador personal le siguieron múltiples innovaciones de software y hardware, incluyendo la denominada

\footnotetext{
3 Ver pág. 42 del autor [17].
}

interfaz, aporte realizado en 1984 por la compañía Xerox, en Palo Alto, Estados Unidos.

Es más, al diseño del microordenador, le prosiguió el diseño del software. El protagonista del software fue Bill Gates y Paul Allen. Ellos buscaron adaptar el programa BASIC para que funcionara en la máquina Altair de Ed Roberts ya diseñada en 1976. Bill Gates y Paul Allen, una vez comprendieron y visionaron el gran potencial tecnológico y económico tanto del computador como del software fundaron Microsoft. Con el desarrollo del software se proporcionaron las condiciones fundamentales para la rápida difusión de los microcomputadores. A lo anterior, es importante sumar la fuerte capacidad de interconexión entre computadores que logró darse con el nacimiento de la agencia ARPA (Advance Research Proyect Agency) en 1969, en donde un grupo de científicos lograron interconectar por medio de la red telefónica varias computadoras y transmitir mensajes escritos [1].

La sorprendente versatilidad y posibilidad de añadir memoria de procesamiento a las computadoras de manera individual o compartiéndola elevó el potencial informático en una red electrónica. Este hecho cambió y transformó la conceptualización y uso de los ordenadores en forma de red, a partir de la década de 1990. Lográndose así el uso y procesamiento de datos en forma interactiva entre computadoras, con potencial de crecimiento exponencial. En donde la disponibilidad de datos, información, velocidad de procesamiento y los bajos costos fueron los protagonistas.

Es destacable que, muchos otros elementos complementarios se dieron en la misma época, por ejemplo el avance de las telecomunicaciones en la misma década de 1970. Las telecomunicaciones facilitaron la capacidad de interconexión y de procesamiento de datos e información. Acciones que se lograron por las sinergias de innovación entre los dispositivos microelectrónicos, así como la capacidad de la informática, la disponibilidad y la misma facilidad de las telecomunicaciones. Sinergias tecnológicas que revolucionaron el contexto de los microordenadores y de las TIC. Hechos que condujeron a la aparición de Internet, como un proceso de maduración durante varias décadas, gracias a la interrelación de computadoras y teléfonos [1]. Internet inicialmente surgió en un 
contexto científico-militar y privado, pero alrededor de 1995 quedó disponible a la sociedad global.

De este modo, en términos de Snow y Brynjolfsson, la sistemática aparición de las TIC dio lugar a un enorme proceso global de "la más grande revolución tecnológica que el hombre haya conocido ${ }^{4 "}$ " $[11,42]$. En donde su surgimiento en sí mismo ha pasado por múltiples etapas desde su discusión como la paradoja de la productividad, la evidencia como un factor determinante de los actuales acelerados niveles de productividad internacional, hasta el factor determinante de la innovación.

No obstante, mientras para algunas regiones geográficas y países la acelerada expansión y uso de las TIC no se ha detenido, para América Latina y otras pocas regiones en desarrollo su difusión se ha mostrado lenta. Convirtiéndose en un paradigma tecnológico, económico y conceptual en los términos de Kuhn [32].

\section{METODOLOGÍA}

El presente estudio metodológicamente manejó las técnicas de factorial y clúster ${ }^{5}$. La técnica utilizó el análisis de varianza simple ANOVA con la prueba $\mathrm{F}$ [22-23, 36, 50], empleando la varianza y las medias estadísticas. La metodología empleó como variables proxy: teléfono fijo por cada 100 habitantes, uso de Internet por cada 100 habitantes y uso de computadores personales (PC) por cada cien habitantes utilizados, aquí se retomó el diseño metodológico de Quiroga-Parra [40]. Dichas variables actuaron como variables proxy de las tecnologías de la información y la comunicación en el presente caso y variables independientes del objetivo planteado.

La ecuación (1) muestra el modelo estadístico de clúster o conglomerados aleatorizados, utilizada como técnica central en este trabajo.

$$
Y_{i j}=\mu+\tau_{i}+\beta_{j}+\varepsilon_{i j}
$$

\footnotetext{
4 Ver pág. 3 del autor [42].

5 El análisis factorial como técnica estadística busca reestructurar una serie de variables originales, en nuevas variables que expresen patrones o relaciones complejas subyacentes en el conjunto de datos originales, y que serían difícilmente percibidas al analizar las variables individualmente. Ver J. Hair, R. Anderson, R. Tatham y W. Black, 2008 [50].
}

En donde la variable dependiente $\left(Y_{i j}\right)$ depende de la media total $(\mu)$, del efecto no aleatorio del tratamiento $i\left(\tau_{i}\right)$, del efecto no aleatorio del bloque $j\left(\beta_{j}\right)$ y los términos de error aleatorios $\left(\varepsilon_{i j}\right)$. Así, el modelo ANOVA permitirá medir la significancia estadística de las diferencias de las medias aritméticas de los grupos determinados por los países de América Latina y, a su vez, establecidos por las variables dependientes proxy de las TIC mencionadas.

Las técnicas estadísticas utilizadas fueron parte de una fase de exploración de las TIC en su uso. Aspecto considerado desde la perspectiva de los hechos de producción con inferencia estadística, antes que de razones causales, elementos que se reflejan en los tres períodos estudiados. La unidad de análisis fue América Latina, con una muestra de 22 países: Argentina (ARG), Bolivia (BOL), Chile (CHL), Colombia (COL), Panamá (PAN), República Dominicana (DOM), República Bolivariana de Venezuela (VEN), Costa Rica (CRI), Ecuador (ECU), El Salvador (SLV), Guatemala (GTM), Honduras (HND), Nicaragua (NIC), Paraguay (PRY), Brasil (BRA), Cuba (CUB), Guyana (GUY), Jamaica (JAM), México (MEX), Perú (PER), Trinidad y Tobago (TTO) y Uruguay (URY).

Los datos fueron tomados directamente de las bases de datos del Banco Mundial, ITU y la OECD, para los períodos 2000, 2006 y 2008. El diseño utilizado correspondió a una metodología cuantitativa-cualitativa con los datos de los tres períodos señalados, y soportado por la literatura de frontera disponible a la fecha del estudio, sobre el problema objeto de análisis. El diseño garantizó un eficaz e importante grado de control, que aportó a la validez interna del trabajo. El grado de dificultad radicó en la disponibilidad de datos de algunos países.

Para la selección de los tres años se tomó en consideración que, el año 2000 fue el inicio del auge del uso de las TIC en los países desarrollados. Los dos siguientes años se tomaron con base en la observación del comportamiento empírico de estas tecnologías en América Latina, y la disponibilidad de datos estadísticos.

La metodología describió el comportamiento estadístico y gráfico de las TIC, en forma de clúster (conglomerados) en los diferentes países 
de la región, por medio de las tres variables proxy indicadas en estas tecnologías. Señalando a las TIC en este caso como un factor determinante de los hechos de producción, que afectan los niveles de productividad y crecimiento [11-13].

\section{RESULTADOS}

Para el análisis de las TIC en América Latina inicialmente se tomaron 21 variables que fue preciso reducir a tres. Para hacer la reducción de variables, se utilizó la técnica de factoriales. Posteriormente para agrupar los países por sus características tecnológicas se hizo uso de la técnica de los clústeres. La técnica de factorial hace uso de la matriz de correlaciones, y del método de componentes principales, en donde se tomó un número fijo de factores igual a 2 y rotación varimax, utilizando cargas factoriales con regresiones, manejando las pruebas de adecuación, como el KMO y la de Bartlett. De esta manera, se obtuvo como resultado los tres nuevos factores representativos de las variables observadas. Estos se escogieron de acuerdo a la varianza total explicada por cada componente, de las cargas factoriales de cada variable en cada componente y de las comunalidades respectivas [22-23].

A su vez, los clústeres o conglomerados como técnica de análisis multivariante consiste en distinguir individuos o elementos que compartan semejanzas entre sí y agruparlos. De manera que, los individuos dentro de cada grupo sean lo más homogéneos posible pero heterogéneos entre ellos; cada elemento pertenece a un único grupo o subconjunto. El propósito primordial es definir una estructura de datos colocando las observaciones similares en grupos [22-23, 50]. La clasificación de los individuos en grupos permite estudiar la estructura subyacente de los datos y las relaciones, determinando las variables que poseen mayor poder de clasificación y observar que características comparten los individuos de cada grupo.

\section{Clúster TIC América Latina 2000}

El análisis de clúster para los 22 países de América Latina estudiados, con los componentes de TIC (uso Internet por cada 100 habitantes, uso teléfono por cada 100 habitantes y uso del PC por cada 100 habitantes) para el año 2000 se muestra en la Tabla 1. Los datos expresan la distribución de los países de América Latina en tres clústeres diferenciados $(1,2,3)$ y su comportamiento estadístico. En la cuarta columna presenta las tres variables proxis de tecnologías de la información y la comunicación utilizadas. En las columnas siguientes se observa la ANOVA, con su prueba estadística y su grado de significancia. En las tres últimas columnas se presentan las medias estadísticas y las varianzas de cada uno de los clústeres.

En la primera parte de la Tabla 1 despliega el clúster 1, compuesto por Chile, Costa Rica, Uruguay y Trinidad Tobago. El clúster número 2 lo conforman Bolivia, Cuba, Ecuador, El Salvador, Guatemala, Guyana, Honduras, Nicaragua, Paraguay, Perú, República Dominicana y Venezuela. En el clúster 3 se encuentran Argentina, Brasil, Colombia, Jamaica, México y Panamá.

La segunda parte de la Tabla 1 muestra las tres variables TIC utilizadas, producto del proceso de aplicar factoriales. En la parte 3 de la tabla se observa del estadístico $\mathrm{F}$ y el p-valor con un valor de cero para todas las variables, denotando que estas son

Tabla 1. Resumen clúster TIC para América Latina, año 2000.

\begin{tabular}{|c|c|c|c|c|c|c|c|c|}
\hline \multicolumn{3}{|c|}{ Clústeres } & \multirow{2}{*}{ Variables TIC } & \multicolumn{2}{|c|}{ Anova } & \multicolumn{3}{|c|}{ Estadísticos descriptivos } \\
\hline 1 & 2 & 3 & & $\mathbf{F}$ & Sig. & Clúster $1^{6}$ & Clúster 2 & Clúster 3 \\
\hline CHL & $\begin{array}{l}\text { BOL, CUB } \\
\text { ECU, SLV }\end{array}$ & $\begin{array}{l}\text { ARG } \\
\text { BRA }\end{array}$ & $\begin{array}{l}\text { Uso Internet por cada } 100 \\
\text { habitantes }\end{array}$ & 14,931 & 0,000 & $\begin{array}{l}\overline{\mathrm{X}}=10,139 \\
\sigma=4,655\end{array}$ & $\begin{array}{l}\overline{\mathrm{X}}=2,105 \\
\sigma=1,847\end{array}$ & $\begin{array}{l}\overline{\mathrm{x}}=4,488 \\
\sigma=2,052\end{array}$ \\
\hline $\begin{array}{l}\text { CRI } \\
\text { URY }\end{array}$ & $\begin{array}{l}\text { GTM, GUY } \\
\text { HND, NIC }\end{array}$ & $\begin{array}{l}\text { COL } \\
\text { JAM }\end{array}$ & $\begin{array}{l}\text { Uso teléfono fijo por cada } 100 \\
\text { habitantes }\end{array}$ & 63,481 & 0,000 & $\begin{aligned} \bar{x} & =24,202 \\
\sigma & =2,889\end{aligned}$ & $\begin{array}{l}\bar{x}=7,215 \\
\sigma=2,625\end{array}$ & $\begin{array}{l}\overline{\mathrm{x}}=17,238 \\
\sigma=3,176\end{array}$ \\
\hline TTO & $\begin{array}{l}\text { PRY, PER } \\
\text { DOM, VEN }\end{array}$ & $\begin{array}{l}\text { MEX } \\
\text { PAN }\end{array}$ & $\begin{array}{l}\text { Uso PC por cada } 100 \\
\text { habitantes }\end{array}$ & 28,876 & 0,000 & $\begin{aligned} \overline{\mathrm{x}} & =10,310 \\
\sigma & =3,795\end{aligned}$ & $\begin{array}{l}\overline{\mathrm{x}}=2,214 \\
\sigma=1,129\end{array}$ & $\begin{array}{l}\overline{\mathrm{x}}=4,933 \\
\sigma=1,275\end{array}$ \\
\hline
\end{tabular}

Fuente: Tomado de Tesis doctoral [40], procesado con datos de WDI, http://ddp-ext.worldbank.org/ext/DDPQQ/member. do?method=getM. 
estadísticamente significativas, e indicando que los clústeres se encuentran claramente diferenciados entre sí por cada uno de los componentes. En la sección derecha de la tabla se presentan los promedios y desviaciones estándar de los 3 clústeres, respecto a cada una de las tres variables TIC. De donde se aprecia que, los países del clúster 1 tienen los valores promedios más altos en todos los componentes TIC. Los del clúster 2 registran los valores promedios más bajos y los países del clúster 3 muestran niveles medios de uso de TIC.

Asimismo, las Figuras 1, 2 y 3, presentan gráficamente la composición y dispersión de los clúster por componentes TIC, para el año 2000. Componentes que se encuentran descritos en los ejes de las ordenadas (Y) y abscisas (X) del plano cartesiano de cada figura definidos en unidades. En donde se observan cada uno los países de la muestra de América Latina, acorde con el clúster al que pertenece.

De manera que, los países de la parte superior (color azul) pertenecen al clúster 1, los de la parte inferior (color verde) al clúster 2 y los de la parte intermedia (color rojo) al clúster 3. La Figura 1 enseña los países por uso de teléfono e Internet, la Figura 2 muestra a quienes usan PC e Internet y la Figura 3 los países que usan PC y teléfono.

El mayor uso del teléfono fijo e Internet por cada 100 habitantes lo tienen Chile, Uruguay, Trinidad-Tobago

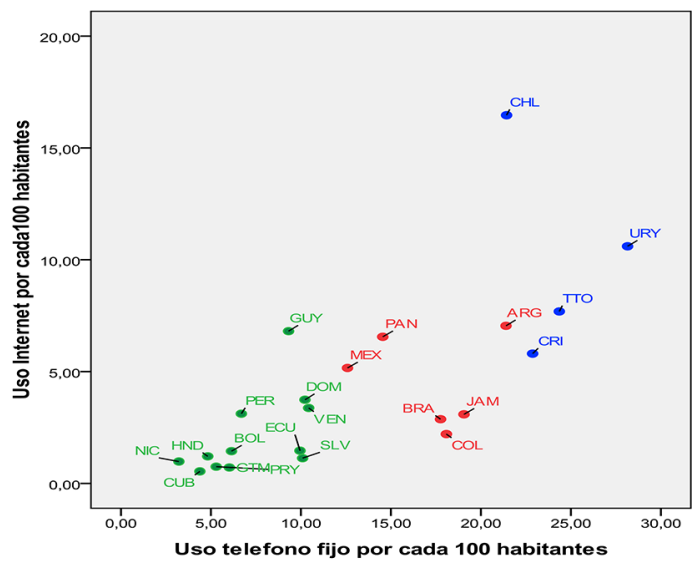

Figura 1. Clúster países de América Latina que usan el teléfono fijo vs. Internet año 2000.

Fuente: Tomado de [40], procesado con datos de WDI, http://ddp-ext.worldbank.org/ext/DDPQQ/ member.do?method=getMembers. y Costa Rica pertenecientes al clúster 1. Algunos de los países con menor uso del teléfono fijo e Internet por cada 100 habitantes en el año 2000 son Nicaragua, Cuba, Paraguay, Salvador y Honduras del clúster 2, ver Figura 1.

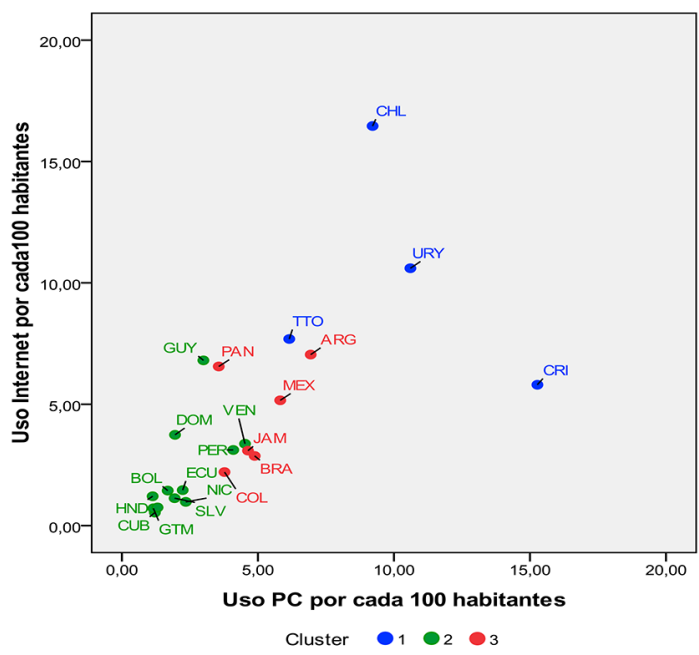

Figura 2. Clústeres países de América Latina que usan el PC vs. Internet, año 2000.

Fuente: Tomado de [40], procesado con datos de WDI, http://ddp-ext.worldbank.org/ext/DDPQQ/ member.do?method=getMembers

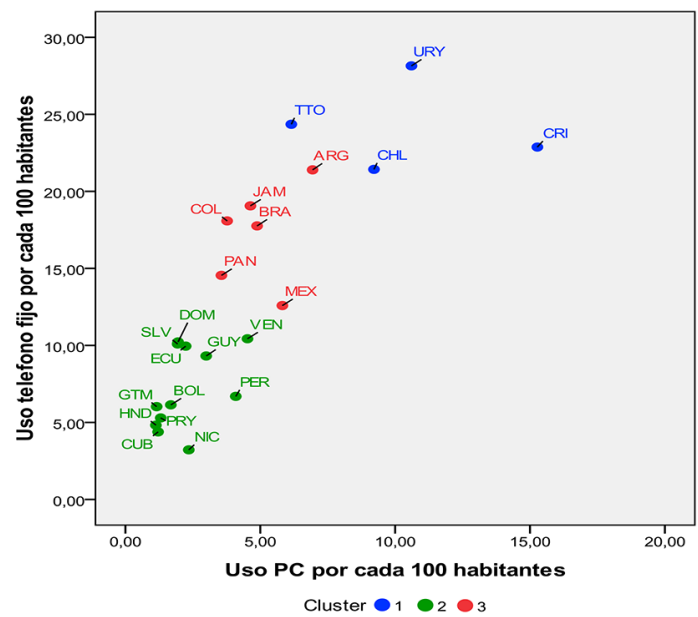

Figura 3. Clústeres países de América Latina que usan PC vs. teléfono, año 2000.

Fuente: Tomado de [40], procesado con datos de WDI, http://ddp-ext.worldbank.org/ext/DDPQQ/ member.do?method=getMembers 
Por otra parte, el uso del PC e Internet por cada 100 habitantes a la fecha, lo lideran en América Latina: Chile, Uruguay, Trinidad-Tobago y Costa Rica del clúster 1, ver Figura 2. Mientras que, algunos de los países con menor uso del PC e Internet por cada 100 habitantes son Honduras, Cuba y Guatemala del clúster 2. Los países de uso de PC e Internet fijo de nivel intermedio, para este período fueron Argentina, México, Panamá, Jamaica, Brasil y Colombia. Este último se muestra en el nivel medio más bajo de este clúster, para el año 2000.

Asimismo, para el año 2000 los países de América Latina que usan más el PC y teléfono por cada 100 habitantes son Uruguay, Costa Rica y Chile del clúster 1 y algunos de los que lo usan en menor grado son Cuba, Paraguay, Nicaragua y Honduras del clúster 2. La Figura 3 igualmente muestra que en el nivel intermedio están: Argentina, Jamaica, Brasil, Colombia, Panamá y México.

De la Tabla 1 se deduce que, en promedio, solo el $10,13 \%$ de los países correspondientes al clúster 1 usan Internet; que el 24,20\% de los países correspondientes al clúster 1 usan el teléfono fijo; y que, el $10,31 \%$ de los países correspondientes al clúster 1 usan el PC, todos por cada 100 habitantes, en el período del año 2000.

Para el clúster 2, solamente el 2,1\% de los países del conglomerado usan Internet, el 7,2\% de estos hacen uso el teléfono fijo y únicamente el 2,21\% de ellos utilizan el PC, todos por cada 100 habitantes.

En relación al clúster 3, por cada 100 habitantes de los países en estudio, el 4,4\% usan Internet, el $17,2 \%$ usan el teléfono fijo y el $4,9 \%$ de ellos utilizan PC.

En suma, de acuerdo con los niveles de intensidad de uso de los componentes TIC en el año 2000, representados en las Figuras 1, 2 y 3, los países que tienen mayor uso en las tres variables ( 2 componentes) son Costa Rica, Uruguay y Chile agrupados en el clúster 1. Mientras que los países con más bajos usos de las variables TIC son Cuba, Nicaragua y Paraguay quienes hacen parte del clúster 2 .

\section{Clúster TIC América Latina 2006}

El avance de resultados del clúster y su conformación para los países de América Latina en los componentes TIC para el año 2006 se muestran en la Tabla 2. Esta presenta igualmente las estadísticas TIC de la región. Los países se exponen en tres clústeres diferenciados $(1,2,3)$ y que, en este sentido señalan su comportamiento estadístico.

En este caso se especifica que el clúster 1 está formado por Jamaica solamente, el que presenta el valor más alto en uso de Internet y valores medios de uso de teléfono fijo y PC para este año.

El clúster 2 lo conforman Bolivia, Colombia, Cuba, Ecuador, El Salvador, Guatemala, Guyana, Honduras, Nicaragua, Panamá, Paraguay, Perú, República Dominicana y Venezuela, quienes se caracterizan por presentar los valores promedios más bajos en todas las variables TIC, ver Tabla 2.

Análogamente, en el clúster 3 se encuentran Argentina, Brasil, Chile, Costa Rica, México, Trinidad, Tobago y Uruguay, que se distinguen por

Tabla 2. Resumen clúster TIC para América Latina, año 2006.

\begin{tabular}{|c|c|c|c|c|c|c|c|c|}
\hline \multicolumn{3}{|c|}{ Clústeres } & \multirow{2}{*}{ Variables TIC } & \multicolumn{2}{|c|}{ Anova } & \multicolumn{3}{|c|}{ Estadísticos descriptivos } \\
\hline 1 & 2 & 3 & & $\mathbf{F}$ & Sig. & Clúster 1 & Clúster 2 & Clúster 3 \\
\hline \multirow{3}{*}{ JAM } & $\begin{array}{l}\text { BOL, COL } \\
\text { CUB, ECU }\end{array}$ & $\begin{array}{l}\text { ARG } \\
\text { BRA }\end{array}$ & $\begin{array}{l}\text { Uso Internet por cada } 100 \\
\text { habitantes }\end{array}$ & 19,953 & 0,000 & $\begin{aligned} \overline{\mathrm{x}} & =48,82 \\
\sigma & =0\end{aligned}$ & $\begin{aligned} \mathrm{X} & =11,633 \\
\sigma & =7,118\end{aligned}$ & $\begin{aligned} \overline{\mathrm{x}} & =23,838 \\
\sigma & =5,331\end{aligned}$ \\
\hline & $\begin{array}{l}\text { SLV, GTM } \\
\text { GUY, HND }\end{array}$ & $\begin{array}{l}\mathrm{CHL} \\
\mathrm{CRI}\end{array}$ & $\begin{array}{l}\text { Uso teléfono fijo por cada } 100 \\
\text { habitantes }\end{array}$ & 21,507 & 0,000 & $\begin{aligned} \overline{\mathrm{x}} & =12,82 \\
\sigma & =0\end{aligned}$ & $\begin{aligned} \overline{\mathrm{x}} & =11,134 \\
\sigma & =4,154\end{aligned}$ & $\begin{aligned} \overline{\mathrm{x}} & =24,066 \\
\sigma & =4,531\end{aligned}$ \\
\hline & $\begin{array}{l}\text { NIC, PAN } \\
\text { PRY,PER, } \\
\text { DOM, VEN }\end{array}$ & $\begin{array}{l}\text { MEX } \\
\text { TTO } \\
\text { URY }\end{array}$ & $\begin{array}{l}\text { Uso PC por cada } 100 \\
\text { habitantes }\end{array}$ & 16,833 & 0,000 & $\begin{array}{l}\overline{\mathrm{x}}=6,70 \\
\sigma=0\end{array}$ & $\begin{array}{l}\bar{x}=5,447 \\
\sigma=3,405\end{array}$ & $\begin{array}{l}\bar{x}=15,350 \\
\sigma=4,277\end{array}$ \\
\hline
\end{tabular}

Fuente: Tomado de [40], procesado con datos de WDI, http://ddp-ext.worldbank.org/ext/DDPQQ/member.do?method=getMembers. 
tener los mejores promedios de los tres grupos en las variables teléfono y de PC ver; sin embargo, para Internet muestran una media de 23,8, Tabla 2.

En la cuarta columna de la Tabla 2 se presentan las tres variables proxis de las TIC utilizadas. En las columnas siguientes se observa la ANOVA, con su prueba estadística y su grado de significancia. En las tres últimas columnas se muestran las medias estadísticas y las varianzas de cada uno de los clústeres. El valor del estadístico $\mathrm{F}$ especifica que los clústeres están bien diferenciados entre sí por cada una de las tres variables TIC, con p-valor significativo al $0,000 \%$. Los valores de los promedios de cada variable y las desviaciones estándares están en la parte derecha de la Tabla 2. De acuerdo con los resultados de la tabla, el 48,8\% de las personas de Jamaica del clúster 1 usan Internet, el 12,8\% usan el teléfono fijo y el 6,7\% usan el PC, todos por cada 100 habitantes.

Con el mismo indicador de variable, el 11,6\% de los países correspondientes al clúster 2 usan Internet. El $11,1 \%$ que conforman este clúster usan el teléfono fijo. $\mathrm{Y}$ el 5,4\% de ellos pertenecientes al mismo clúster usan PC.

En la cuarta columna de la Tabla 2 presenta las tres variables proxis de las TIC utilizadas. En las columnas siguientes se observa la ANOVA, con su prueba estadística y su grado de significancia. En las tres últimas columnas se presentan las medias estadísticas y las varianzas de cada uno de los clústeres.

A la par, los países que conforman el clúster 3 usan Internet en el 23,8\%; el teléfono fijo en el 24,1\%; y el PC en el $15,3 \%$, por cada 100 habitantes, ver Tabla 2.

En este sentido, las Figuras 4, 5 y 6 y resaltan que en el clúster 1 Jamaica, quedó diferenciado en su mayor parte por el uso intensivo variable Internet. De los países que conforman el clúster 3, Costa Rica y Uruguay presentan los mejores niveles de uso de teléfono y Brasil los mejores valores de Internet en su grupo. Análogamente los países que exhiben los valores más bajos de uso de TIC en el año 2006 son Nicaragua, Cuba, Honduras, Paraguay y Bolivia, todos ellos pertenecientes al clúster 2 .

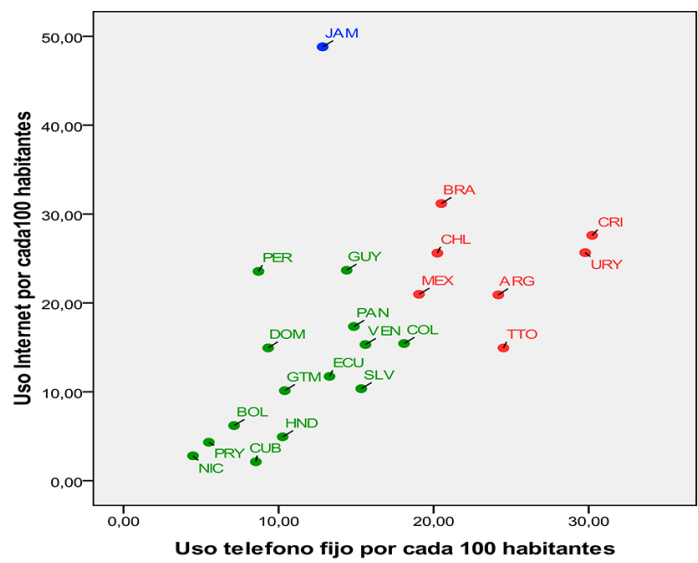

Figura 4. Clúster países de América Latina que usan Internet vs. teléfono, año 2006.

Fuente: Tomado de [40], procesado con datos de WDI, http://ddp-ext.worldbank.org/ext/DDPQQ/ member.do?method=getMembers

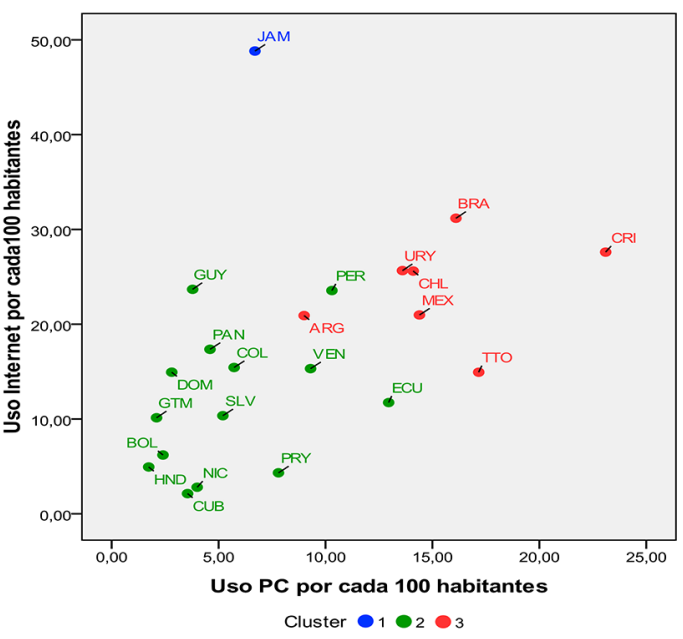

Figura 5. Clústeres países de América Latina que usan Internet vs. uso del PC, año 2006.

Fuente: Tomado de [40], procesado con datos de WDI, http://ddp-ext.worldbank.org/ext/DDPQQ/ member.do?method=getMembers

De la Figura 4 se deduce que el país de América Latina con mayor uso de Internet al año 2006 es Jamaica y los países con menor uso de este al mismo año son Nicaragua, Paraguay y Cuba. Los países con mayor uso de teléfono fijo disponible son Costa Rica y Uruguay. 


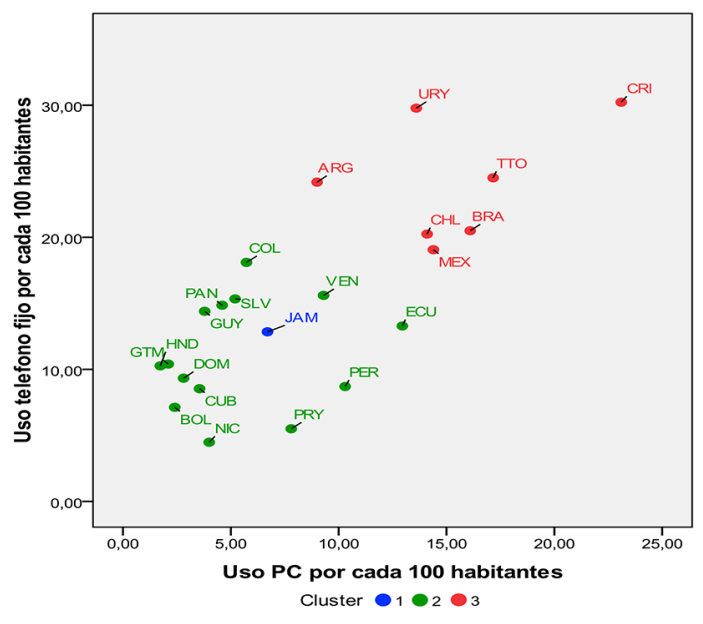

Figura 6. Clústeres países de América Latina que usan teléfono vs. PC, año 2006.

Fuente: Tomado de [40], procesado con datos de WDI, http://ddp-ext.worldbank.org/ext/DDPQQ/ member.do?method=getMemb

A su vez, el país de América Latina con mayor uso de PC y teléfono fijo al año 2006 por cada 100 habitantes es Costa Rica, seguido de Trinidad Tobago y Brasil, ver Figuras 5 y 6. Los gráficos anteriores de dispersión muestran y destacan una relación lineal directa entre las tres variables TIC, es decir que si se da un incremento en una, en la otra también.

\section{Clúster TIC América Latina 2008}

Por otra parte, los resultados del análisis de clústeres realizados en la muestra de 22 países de América Latina para el año 2008 en los componentes TIC se presentan en la Tabla 3. Esta revela que el clúster 1 está formado por Argentina, Brasil, Chile, Costa Rica, México, Trinidad y Tobago, Uruguay y Venezuela. A su vez, Bolivia, Cuba, Ecuador, El Salvador, Guatemala, Guyana, Honduras, Nicaragua, Panamá, Paraguay, Perú y República Dominicana conforman el clúster 2, mientras que en el clúster 3 se ubican Colombia y Jamaica.

En efecto, el clúster 1 se caracteriza por tener los promedios más altos en las variables de uso de teléfono, uso de PC y un promedio de Internet de nivel medio. El clúster 2 muestra los valores promedios más bajos en todas las variables TIC. Mientras que el clúster 3 tiene el promedio más alto en Internet y valores promedios de nivel medio en teléfono y PC. El estadístico F confirma que los clústeres están diferenciados entre sí por cada una de las variables.

La misma Tabla 3 además de presentar la distribución de los países en 3 clústeres diferenciados $(1,2,3)$, muestra su comportamiento estadístico. En la cuarta columna se presentan las tres variables proxis de tecnologías de la información y la comunicación utilizadas. En las columnas siguientes se observa la ANOVA, con su prueba estadística y grado de significancia. En las tres últimas columnas se presentan las medias estadísticas y las varianzas de cada uno de los clústeres.

De acuerdo con los resultados de la Tabla 3, los países que conforman el clúster 1 , por cada 100 habitantes, usan Internet en el 28,5\%, teléfono fijo en el $23,1 \%$, y PC en el 14,3\%.

Tabla 3. Resumen clúster TIC para América Latina, año 2008.

\begin{tabular}{|c|c|c|c|c|c|c|c|c|}
\hline \multicolumn{3}{|c|}{ Clústeres } & \multirow{2}{*}{ Variables TIC } & \multicolumn{2}{|c|}{ Anova } & \multicolumn{3}{|c|}{ Estadísticos Descriptivos } \\
\hline 1 & 2 & 3 & & $\mathbf{F}$ & Sig. & Clúster 1 & Clúster 2 & Clúster 3 \\
\hline $\begin{array}{l}\text { ARG } \\
\text { BRA }\end{array}$ & BOL, CUB & & $\begin{array}{l}\text { Uso Internet por cada } 100 \\
\text { habitantes }\end{array}$ & 16,552 & 0,000 & $\begin{array}{l}\overline{\mathrm{x}}=28,533 \\
\sigma=7,541\end{array}$ & $\begin{array}{l}\overline{\mathrm{x}}=14,100 \\
\sigma=8,211\end{array}$ & $\begin{array}{l}\bar{x}=45,218 \\
\sigma=10,208\end{array}$ \\
\hline $\begin{array}{l}\text { CHL } \\
\text { CRI }\end{array}$ & $\begin{array}{l}\text { ECU, SLV } \\
\text { GTM,GUY }\end{array}$ & $\mathrm{COL}$ & $\begin{array}{l}\text { Uso teléfono fijo por cada } 100 \\
\text { habitantes }\end{array}$ & 22,695 & 0,000 & $\begin{array}{l}\overline{\mathrm{x}}=23,192 \\
\sigma=4,990\end{array}$ & $\begin{array}{l}\bar{x}=10,344 \\
\sigma=3,639\end{array}$ & $\begin{aligned} \bar{x} & =15,654 \\
\sigma & =3,413\end{aligned}$ \\
\hline $\begin{array}{l}\text { MEX } \\
\text { TTO } \\
\text { URY } \\
\text { VEN }\end{array}$ & $\begin{array}{l}\text { HND, NIC } \\
\text { PAN, PRY } \\
\text { PER, DOM }\end{array}$ & JAM & $\begin{array}{l}\text { Uso PC por cada } 100 \\
\text { habitantes }\end{array}$ & 14,688 & 0,000 & $\begin{array}{l}\bar{x}=14,345 \\
\sigma=4,392\end{array}$ & $\begin{array}{l}\bar{x}=5,150 \\
\sigma=3,467\end{array}$ & $\begin{array}{l}\bar{x}=6,802 \\
\sigma=0,074\end{array}$ \\
\hline
\end{tabular}

Fuente: Tomado de [40], procesado con datos de WDI, http://ddp-ext.worldbank.org/ext/DDPQQ/member.do?method=getMembers 
El 14,1\% de los países correspondientes al clúster 2 usan Internet, el 10,3\% de ellos usan el teléfono fijo y el 5,1\% usan el PC; por cada 100 habitantes.

A su vez, por cada 100 habitantes, el 45,2\% de los países correspondientes al clúster 3 usan Internet, el $15,6 \%$ usan el teléfono fijo y el $6,8 \%$ de ellos usan el PC.

En las Figuras 7, 8 y 9 de dispersión se aprecian la composición de los 3 clústeres y la manera como están distribuidos en un plano cartesiano, para el año 2008 en América Latina.

Las Figuras 7 y 8 muestra como Jamaica seguida de Uruguay y Colombia presentan los mejores indicadores de uso de Internet, igual se destacan de manera ascendente de Brasil y Chile. Contrariamente, Nicaragua y Paraguay son los países de la región con los más bajos indicadores de Internet, ver Figura 7. En este caso, Costa Rica se mantiene en el año 2008 como el país de América Latina que más utiliza el PC, seguido de Brasil, Trinidad Tobago, México, Chile y Uruguay.

Colombia, por su parte, se ubicó en un punto de uso, alrededor de 7 PC por cada 100 habitantes, al igual que Jamaica. De su parte, Honduras, Guatemala y Bolivia son los de menor uso de PC, ver Figura 8.

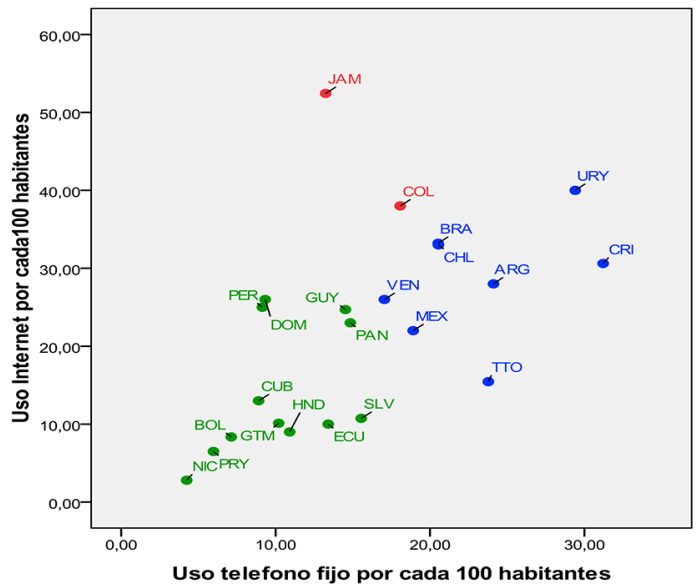

Figura 7. Clústeres países de América Latina que usan Internet vs. teléfono, año 2008.

Fuente: Tomado de [40], procesado con datos de WDI, http://ddp-ext.worldbank.org/ext/DDPQQ/ member.do?method=getMembers.

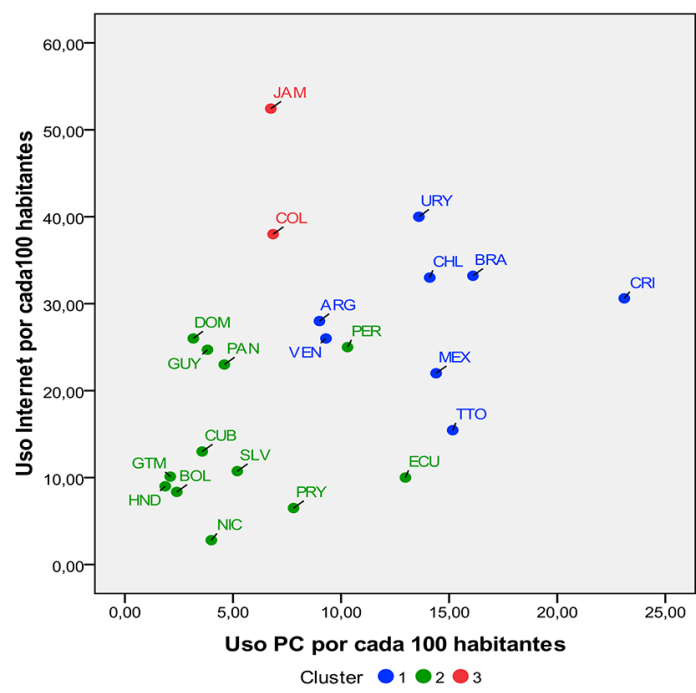

Figura 8. Clúster países de América Latina que usan TIC al 2008. Uso internet vs. PC, año 2008.

Fuente: Tomado de [40], procesado con datos de WDI, http://ddp-ext.worldbank.org/ext/DDPQQ/ member.do?method=getMembers

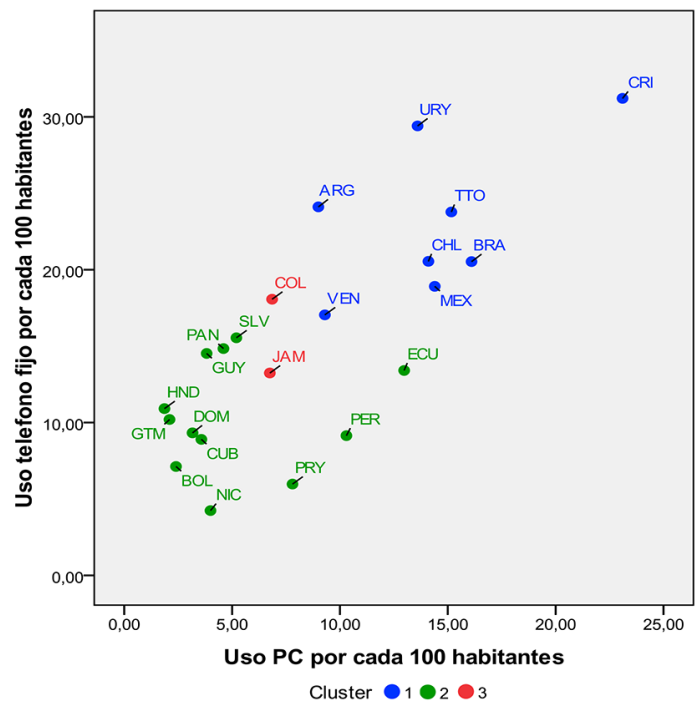

Figura 9. Clúster países de América Latina que usa teléfono vs. PC, año 2008.

Fuente: Tomado de [40], procesado con datos de WDI, http://ddp-ext.worldbank.org/ext/DDPQQ/ member.do?method=getMembers.

Los datos estadísticos y las figuras muestran que, los países con mayor uso de teléfono fijo en América Latina para el año 2008 fueron Costa Rica y Uruguay 
observándose una consistencia en su infraestructura TIC, frente el avance del uso de Internet.

Le siguen Argentina, Trinidad Tobago Chile y Brasil, para el año 2008. Colombia se muestra en un punto medio bajo, alrededor de 18 teléfonos por 100 personas. Con respecto a, Nicaragua, Bolivia y Paraguay se presentan con los indicadores más bajos en el uso de teléfonos fijos por 100 personas en América Latina, ver Figura 7. Es de anotar que, los teléfonos fijos como infraestructura tecnológica que son relevantes para la expansión de la banda ancha de Internet.

En síntesis, los resultados del análisis clúster en América Latina, para las variables TIC durante los tres años se señala que Chile, Costa Rica, Uruguay, Brasil y Trinidad y Tobago, muestran niveles constantes de crecimiento. Jamaica a pesar del importante uso que hace de Internet, no revela crecimiento en el uso de PC. Aspecto similar ocurre con Colombia en el último período.

El trabajo desde la perspectiva teórica y de los resultados empíricos, muestra un importante aporte como nuevo conocimiento a la necesidad del uso de las TIC en América Latina. En concreto en su aplicación a la innovación y la productividad. Mostrando la manera cómo la región no ha logrado asimilar totalmente el concepto de las TIC y su aplicación, en el acelerado avance global de la economía del conocimiento, centrada esta en el uso intensivo de la información y el conocimiento.

\section{DISCUSIÓN ACADÉMICA}

Los resultados empíricos para el año 2000 en América Latina muestran que, el uso de Internet y de PC son relativamente bajos, en relación con los trabajos empíricos de los países desarrollados [2627]. Aspecto a señalar como primeras evidencias del relativo bajo uso de TIC en la región, en el inicio de la década.

Asimismo se observa un uso bajo de teléfonos fijos en el mismo período. De hecho, el bajo nivel de disponibilidad de teléfonos fijos ha dificultado el avance de Internet y del mismo PC. Observándose que América Latina desde el mismo período del año 2000 no presenta la suficiente infraestructura tecnológica que facilite el avance acelerado de las
TIC; como posible hecho empírico que apunta a la verificación de la hipótesis planteada, de que los niveles de uso de TIC en América Latina son relativamente bajos. Aspecto que se muestra en la dirección de los trabajos de Brynjolfsson y Hitt [13-15].

Los países con crecientes avances de las TIC en los tres períodos estudiados como Chile, Brasil, Uruguay y Costa Rica pueden ser quienes mejor puedan acelerar el uso y velocidad de la banda ancha. Hecho que, a su vez activaría el potencial de mejoramiento de la productividad; sin embargo, esta probabilidad no se muestra muy alta, dada la baja infraestructura de teléfonos fijos que facilite su aceleramiento. Paralelamente los resultados del uso de PC en América Latina se observa en niveles relativamente bajos, factor que puede estar frenando los potenciales de innovación y productividad. Resultados científicos que contrastan con los hallazgos en [30,40], y ciertamente en la dirección de los estudios de Brynjolfsson [11, 14].

Casos como los de Colombia se infiere que, mejoró positivamente la infraestructura de Internet en los dos últimos períodos. Sin embargo, el bajo uso de PC dificultan el acceso a la información y por lo tanto al conocimiento, y sistemáticamente el mejoramiento de la innovación y la productividad. Específicamente por la curva de aprendizaje, la cual toma tiempo para estabilizarse e incidir en la productividad en los términos de los estudios de Arvanitis y Loukis, Badescu y Garcés [3-4, 6].

Los resultados empíricos muestran que, los países más desarrollados de América Latina como Chile y Brasil, presentan avances importantes en el uso de TIC. Sin embargo, desde la perspectiva de la literatura internacional del tema y de los trabajos similares realizados en los países desarrollados de la OECD, se observa una brecha tecnológica importante frente a los mejores avances en la región. Lo anterior desde la perspectiva teóricocientífico de DeLong [19], Black y Lynch [9] y Stiroh [41].

Si bien es cierto que, en América Latina los resultados estadísticas muestran algunos avances en el uso de TIC. No obstante, la paradoja de la TIC de Solow [37] se muestra vigente y por comprender, para que los resultados se puedan ver reflejados en la productividad. Y, científicamente poco se 
muestran en la dirección de los planteamientos de Brynjolfsson y Hitt $[11,13]$. De hecho, el estudio de los tres períodos de América Latina, lleva a la verificación de la hipótesis previamente planteada.

En virtud de lo anterior, se observa la importancia de proyectar a futuro líneas de investigación en América Latina en esta misma perspectiva.

\section{CONCLUSIONES}

Los resultados del estudio teórico y empírico muestran que América Latina presenta ineficiencias en el conocimiento, uso y aplicación de las TIC. La metodología utilizada permitió agrupar los veintidós países estudiados en tres clústeres diferenciados en sus características tecnológicas. El uso y aplicación de estas tecnologías se expresa marginal para la mayoría de los países.

Las ineficiencias de uso y aplicación de las TIC señalan una importante brecha digital, frente a los estudios internacionales previos de países desarrollados, en el contexto micro y macroeconómico.

El estudio expresa que, la presencia y uso de las TIC en América Latina para el inicio de la década del año 2000 presenta cifras estadísticas modestas. En un importante número de países se observan con bajos niveles de TIC que no superan las cinco unidades por cada cien habitantes. No obstante que, la región en los períodos 2006-2008 muestra sustanciales avances; sin embargo, estos sugieren estar lejos de los indicadores de los estudios científicos internacionales sobre el tema en los países desarrollados. Los resultados son relevantes para el direccionamiento de las políticas públicas en América Latina.

Se infiere que, desde la literatura científica actual, el lento progreso de las TIC en América Latina puede estarse dando por la insuficiente infraestructura tecnológica existente. Igualmente, por el desconocimiento de las instituciones y de los gobiernos de estos países, respecto a la importancia de estas tecnologías digitales transversales en la economía de la región $[2,40]$. Hechos que se evidencian en el insuficiente interés académico y político sobre el tema, y la existencia mínima de estudios de este tipo en la región.
Algunos de los aportes del estudio son: una contribución teórica-empírica al conocimiento de las TIC en la región; una metodología de reducción del alto número de variables a unas pocas representativas; una clasificación de los países en conglomerados de características tecnológicas similares; Un aporte a la literatura internacional del uso de las TIC y a la economía del conocimiento.

Los resultados empíricos y teóricos señalan la presencia de la paradoja de la productividad en América Latina. Hecho que puede estar marcando la existencia de un paradigma tecnoeconómico y social en la región, esto es, en la manera de percibir el potencial de la economía del conocimiento en estos países, por medio del uso de la información en las TIC.

Los bajos usos de Internet y PC pueden estar afectando los indicadores de productividad e innovación de la región. Es así como, bajo la perspectiva de los trabajos empíricos internacionales y literatura sobre TIC, tanto en el contexto macroeconómico, como en las empresas, los resultados de estos hechos de productividad se asimilan a unas primeras evidencias empíricas, por confirmar matemáticamente.

Finalmente, la contrastación teórico-empírica de los resultados de los hechos de productividad de las TIC, permite intuir dificultades en los procesos futuros de innovación y productividad en América Latina. Surgiendo la necesidad de incorporar las TIC a los procesos productivos y de innovación. Hechos que al no ser corregidos oportunamente con política pública puede afectar el crecimiento y desarrollo de la región. Postergando la incorporación de la región a la economía global de la información y el conocimiento. Al igual que, la posibilidad de incrementarse la brecha digital, económica, social y del conocimiento entre la región y los países industrializados.

\section{REFERENCIAS}

[1] J. Abbate. "Inventing the Internet". MIT Press. Cambridge, MA, USA. 1999. ISBN: 0-262-01172-7.

[2] D. Acemouglu y A. Robinson. "Por qué fracasan los países". Deusto. New York, USA. 2013. ISBN: 0-262-01172-7.

[3] S. Arvanitis. "Computerization, workplace organization, skilled labour and firm productivity: evidence for the Swiss business 
sector". Economics of Innovation and New Technologies. Vol. $14 \mathrm{~N}^{\circ}$ 4, pp. 225-249. 2005. ISSN: 1043-8599.

[4] S. Arvanitis and E. Loukis. "Information and communication technologies, human capital, workplace organization and labour productivity: a comparative study based on firm-level data for Greece and Switzerland". Information Economics y Policy. Vol. 21 $\mathrm{N}^{\mathrm{o}}$ 1, pp. 43-61. 2009. ISSN: 0167-6245.

[5] A. Atkeson and P. Kehoe. "The transition to a new economy after the second industrial revolution". NBER Working Paper, pp. 8676. 2001. ISSN: 2194-6108.

[6] M. Badescu and C. Garcés "The impact of information technologies on firm productivity: empirical evidence from Spain". Technovation. Vol. $22 \mathrm{~N}^{\circ}$ 2, pp. 122-129. 2009. ISSN: 0166-4972.

[7] T. Bauer and S. Bender. "Technological change, organizational change, and job turnover". Labour Economics. Vol. 11, pp. 265-291. 2003. ISSN: 0927-5371.

[8] S. Black and L. Lynch. "How to compete: the impact of workplace practices and information technology on productivity". Review of Economics and Statistics. Vol. 83 $\mathrm{N}^{\circ}$ 3, pp. 434-445.2001. ISSN: 0034-6535.

[9] S. Black and L. Lynch. "Measuring organizational capital in the new economy". CES Working Paper, pp. 02-04. Washington D.C., USA. 2002. ISSN: 2067-7693.

[10] E. Brynjolfsson and S. Yang. "Information technology and productivity: a review of the literature". Advances in computers. Vol. 43, pp. 179-214. 1996. ISBN: 978-0-12-012143-4.

[11] E. Brynjolfsson. "The Productivity Paradox of Information Technology". Communications of the ACM. Vol. $35 \mathrm{~N}^{\circ} 12$, pp. 66-77. 1993. ISSN: 0001-0782.

[12] T. Bresnahan, E. Brynjolfsson and L. Hitt. "Information technology, workplace organization, and the demand of skilled labor: firm-level evidence". Quarterly Journal of Economics. Vol. 117, pp. 339-376. 2000. ISSN: 0033-5533.

[13] E. Brynjolfsson and L. Hitt. "Beyond the productivity paradox: computers are the catalyst for bigger changes". Communications of the ACM. Vol. $41 \mathrm{~N}^{\circ}$ 8, pp. 49-55. 1998. ISSN: 0001-0782.
[14] E. Brynjolfsson and L. Hitt. "Beyond computation: information technology, organizational transformation and business performance". Journal of Economic Perspectives. Vol. 14, pp. 23-48. 2000. ISSN: 0895-3309.

[15] E. Brynjolfsson and L. Hitt. "Computing productivity: firm-level evidence". Review of Economics and Statistics. Vol. $85 \mathrm{~N}^{\circ} 4$, pp. 793-808. 2003. ISSN: 0034-6535.

[16] N. Crafts. "The Solow productivity paradox in historical perspective. Long-Term Trends in the World Economy". Copenhagen: University of Copenhagen, pp. 1-22. 2000. URL: http://www.j-bradford-delong.net/ articles_of_the_month/pdf/newsolow.pdf. Fecha de consulta: 01 Diciembre 2015.

[17] M. Castells. "The rise of the network society". ( $2^{\text {a }}$ ed.). Malden: Backwell Publishers Ltd. 2000. ISBN: 0-631-221-40-9.

[18] P. David. "The dynamo and the computer: a historical perspective on the modern productivity paradox". American Economic Review Papers and Proceedings. Vol. $80 \mathrm{~N}^{\mathrm{o}} 2$, pp. 355-361. 1990. ISBN-13: 978-1405196864.

[19] B. DeLong. "A historical perspective on the new economy". Ponencia presentada en The New Economy Conference. Montreal, Canada. 2001.

[20] M. Kranzberg. "The information age: evolution or revolution?". In: Kranzberg, M. (ed.). Information technologies and social transformation. Washington D.C., USA. National Academy of Engineering. 1985. ISBN: 0-309-03529-5.

[21] C. Freeman and C Pérez. "Structural crises of adjustment, business cycles and investment behavior. Technical change and economic theory". In DOSI In technical change and economic theory. Londres: Pinter Publishers. 1988. ISBN-10: 086187949X and ISBN-13: 978-0861879496.

[22] W.C. Guenther. "Analysis of Variance". Prentice Hall, Inc., Englewood Cliffs, Biometrical Journal. Vol. $8 \mathrm{~N}^{\mathrm{o}} 3$, pp. 209. 1966. DOI: 10.1002/bimj.19660080313. URL: http://onlinelibrary.wiley.com/ doi/10.1002/bimj.19660080313/citedby. Fecha Consulta: 10 de diciembre 2015.

[23] H. Scheffé. "The analysis of variance". New York, USA. Wiley Interscience. 1999. ISBN-13: 978-0471345053. 
[24] T. Hempell. "Does Experience Matter? Innovations and the Productivity of ICT in German Services". Centre for European Economic Research (ZEW), Mannheim, pp. 1-28. 2002. URL: ftp://ftp.zew.de/pub/ zew-docs/dp/dp0243.pdf. Fecha de Consulta: 10 de diciembre 2015.

[25] T. Hempell. "Do computers call for training? firm-level evidence on complementarities between ICT and human capital investments". ZEW Discussion Paper No 03-20, Mannheim, pp. 1-43. 2003. URL: ftp://ftp.zew.de/pub/ zew-docs/dp/dp0320.pdf. Fecha de Consulta: 10 de diciembre 2015.

[26] D. Jorgenson and K. Stiroh. "Raising the speed limit: U.S. economic growth in the information age". Brookings Papers on Economic Activity. Vol. 1, pp. 125-211. 2000. ISSN: 0007-2303.

[27] D. Jorgenson. "Information technology and the U.S. economy". American Economic Review. Vol. $9 \mathrm{~N}^{\circ}$ 1, pp. 1-32. 2001. ISSN: 0002-8282.

[28] D. Jorgenson. "Information technology and the G7". Revue de l'OFCE, Vol. $97 \mathrm{~N}^{\circ}$ 5, pp. 189-215. 2006. ISBN: 9782724630664. DOI: $10.3917 /$ reof.073.0189.

[29] D. Jorgenson and K. Vu. "Information technology and the world economy". Scandinavian Journal of Economics. Vol. 1078 № 4, pp. 631-650. December, 2005. ISSN: 0347-0520.

[30] D. Jorgenson and K. Vu. "Latin America and the world economy". Proyecto Sociedad de la Información, Santiago Chile, Economic Commission for Latin America and the Caribbean (ECLAC). 2007. URL: http:// scholar.harvard.edu/files/jorgenson/files/ latinamericaworldeconomy_07_1029.pdf. Fecha de Consulta: 10 de diciembre 2015.

[31] D. Jorgenson, M. Ho and K. Stiroh. "Productivity: Information technology and the American growth resurgence". Vol. 3. The MIT Press. Cambridge, USA. 2005. ISBN: 0-262-10114-4.

[32] T. Kuhn. "La estructura de las revoluciones científicas". México D.F.: Fondo de Cultura Económica. 1971. ISBN: 950·557-113-5.

[33] J. López, B. Minguela, A. Rodríguez and F. Santulli. "Is the internet productive? A firm-level analysis". Technovation. Vol. 26 No 7, pp. 821-826. 2006. ISSN: 0166-4972.
[34] J. Mokyr. "The economics of the industrial revolution". Totowa: Rowman \& Allanheld. 1985. ISBN: 0049410105.

[35] J. Mokyr. "The level of riches: technological creativity and economic progress". Nueva York: Oxford University Press. 1990.

[36] C. Pérez. Modelos econométricos con SPSS. Madrid: Ibergaceta Publicaciones. 2009. ISBN: 8493720860 ISBN-13: 9788493720865.

[37] R. Solow. "We'd better watch out". New York Times Book Review, July 12, 36. 1987. URL: http://www.standupeconomist.com/ pdf/misc/solow-computer-productivity.pdf. Fecha de consulta: 10 de diciembre 2015.

[38] S. Oliner and D. Sichel "The resurgence of growth in the late 1990s: is information technology the story?" Journal of Economic Perspectives. Vol. $14 \mathrm{~N}^{\circ} 4$ fall, pp. 3-22. 2000. ISSN: 0895-3309.

[39] OCDE. "OECD Science, Technology and Industry Outlook". Paris: OCDE. 2001. URL: ISBN 9264195386. http://www.oecd. org/science/inno/2762979.pdf

[40] D. Quiroga-Parra y J. Torrent-Sellens. "Las nuevas fuentes de productividad en América Latina y la OCDE" [online working paper]. (Working Paper Series; WP14-009). IN3 Working Paper Series. IN3 (UOC), pp. 1-34. 2015. ISSN: 20138644. URL: http://journals.uoc.edu/ojs/ index.php/in3-working-paper-series/article/ view/n14-quiroga-parra-torrent-sellens/ n14-quiroga-parra-torrent-sellens. Fecha de consulta: 01 diciembre 2015.

[41] K.J. Stiroh. "Are ICT spillovers driving the new economy?" Review of Income and Wealth, International Association for Research in Income and Wealth. Vol. 48 No $^{\circ}$, pp. 33-57. 2002. ISSN: 1475-4991.

[42] C.P. Snow. "Government Science and Public Policy". Science, Vol. 151, №3711, pp. 650653. 1966. ISSN: 1095-9203. DOI: 10.1126/ science.151.3711.650.

[43] J. Triplett and B. Bosworth. "Productivity in the service sector". Bostworth: Mimeo, The brookings institutions. 2000. ISSN: 1096-2719.

[44] M. Timmer, G. Ypma y B. Van Ark. "IT in the European Union: Driving Productivity Divergence?" [en línea]. GGDC Research Memorandum GD-67, Groningen, University 
of Groningen, pp. 2003. URL: http://citeseerx. ist.psu.edu/viewdoc/download?doi=10.1.1. 462.6011\&rep=rep1\&type $=$ pdf. Fecha de consulta: 10 diciembre 2015.

[45] Timmer, Marcel, Gerard Ypma and B. Van Ark. "Industry-of-Origin Prices and Output PPPs: A New Dataset for International Comparisons, Groningen Growth and Development Centre" (forthcoming). 2005. URL: http://www.euklems.net/meetings/ cm_helsinki/van_ark\&timmer.pdf. Fecha de consulta: 10 diciembre 2015.

[46] J. Torrent y P. Ficapal. "TIC, coinnovación y productividad empresarial: evidencia empírica para Cataluña y comparación internacional". Revista de Economía Mundial. Vol. 6, pp. 1-24. 2010.

[47] Unión Europea. "The impact of the e-economy on European enterprises: economic analysis and policy implications". Communication from the Commission to the Council and the European Parliament. 2001. URL: http://ec.europa.eu/transparency/regdoc/ rep/1/2001/EN/1-2001-711-EN-F1-1.Pdf. Fecha de consulta: 10 diciembre 2015.

[48] J. Van Reenen, M. Draca and R. Sadun. "Productivity and ICTs: a review of the evidence". En: Mansell, R.; Avergerou, C.; Quah, D. y Silverstone, R. (ed.). The Oxford handbook of information and communication technologies, pp. 100-147. University Press. Oxford, USA. 2007. ISBN 9780199266234.

[49] J. Vilaseca y J. Torrent. "TIC, conocimiento y crecimiento económico. Un análisis empírico, agregado e internacional sobre las fuentes de la productividad". Economía Industrial. Vol. 360, pp. 41-60. 2006. ISSN: 0422-2784.

[50] J. Hair, R. Anderson, R. Tatham y W. Black. "Análisis multivariante". Pearson Educación S.A. Madrid, España. 2008. ISBN: 978-84-8322-035-1. 